\title{
ORIGINAL RESEARCH \\ Comparison of Low-Dose With Standard-Dose Multidetector CT in Cervical Spine Trauma
}

\section{T.H. Mulkens \\ P. Marchal \\ S. Daineffe \\ R. Salgado \\ P. Bellinck \\ B. te Rijdt}

B. Kegelaers

J.-L. Termote
BACKGROUND AND PURPOSE: The purpose of this work was to evaluate the possible use of low-dose multidetector CT (MDCT) in cervical clearance of patients with blunt trauma.

MATERIALS AND METHODS: A total of 191 patients underwent cervical spine MDCT with 6- and 16-MDCT: standard-dose $(n=51)$ and low-dose MDCT with tube current modulation at high $(n=70)$ and low ( $n=70$ ) tube voltage (kilovolts). Effective dose, image noise, and subjective image quality were calculated in all of the patients.

RESULTS: MDCT found 18 patients (9.4\%) with a cervical spine fracture, 3 in the standard-dose and 15 in the low-dose group, 14 of them with unstable lesions. Tube current modulation reduced the dose by $50 \%-61 \%$ in all of the low-dose examinations. The mean effective dose was $3.75,1.57$, and $1.08 \mathrm{mSv}$, and mean image noise was 14.82, 17.46, and 19.72 Hounsfield units for standard dose and low dose with high and low kilovolt examinations, respectively. These differences in mean effective dose and image noise were significant between the 3 examination groups (Kruskal-Wallis test: $P<.0001$ and $P=.0001$ ). Evaluation of subjective image quality by 2 radiologists and 2 residents showed no significant difference in image quality score among the 3 examination groups (Kruskal-Wallis tests, $P=.61, .32, .18$, and .31). All of the reviewers correctly detected 18 fractures, except 1 resident, who missed 3 fractures.

CONCLUSION: Low-dose cervical spine MDCT in patients with blunt trauma gives a substantial dose reduction of $61 \%-71 \%$, compared with standard-dose MDCT, with a small increase in image noise and without difference in subjective image quality evaluation.
$\mathbf{R}$ ecent advances have markedly enhanced the clinical applications of CT, especially since the introduction of the spiral scan technique and the subsequent further development of multidetector CT (MDCT) technology. ${ }^{1}$ In this way, MDCT has become the preferred initial imaging technique in the evaluation of patients with acute blunt cervical spinal trauma: a number of reasons make MDCT a better choice over standard radiography, but the main reasons are accuracy, speed, and reduced patient manipulation. ${ }^{2}$

Multiple recent studies add to a growing body of evidence that MDCT should replace standard radiography in patients with blunt trauma who require radiographic evaluation of the cervical spine and in unconscious patients with severe head trauma, whereby standard radiography becomes unnecessary. ${ }^{3-10}$ But only a small number of these patients will have a significant cervical spine injury (CSI): there is only a $2 \%-6 \%$ incidence of significant cervical spinal injury in the symptomatic population after acute trauma. ${ }^{2,11,12}$ Therefore, most of these patients, many being young and otherwise healthy persons, will undergo a much larger radiation dose for their cervical spine clearance. ${ }^{4,13}$ Simply replacing plain films with MDCT creates a dose problem. Moreover, with the presence of MDCT, clinical requests in trauma patients for complete examination of the cervical spine with CT increases, and this changing trend results in a significantly higher radiation dose, especially to nearby radiosensible organs: the thyroid, lens, and breasts. ${ }^{13}$

The purpose of this study was to evaluate the hypothesis that low-dose cervical spine MDCT, using tube current modulation and lower-tube voltage settings, can be used as a screening tool for

Received December 21, 2006; accepted after revision January 27, 2007.

From the Departments of Radiology (T.H.M., P.M., S.D., R.S., P.B., J.-L.T.) and Neurosurgery (B.t.R., B.K.), Heilig Hart Ziekenhuis, Lier, Belgium.

Please address correspondence to Tom H. Mulkens, Department of Radiology, Heilig Hart Ziekenhuis, Mechelsestraat 24, B 2500 Lier, Belgium; e-mail: tom.mulkens@hhzhlier.be DOI 10.3174/ajnr.A0608 cervical spine clearance in patients with blunt trauma with clinical suspicion of CSI and in unconscious trauma patients in which reliable clinical assessment is not possible.

\section{Materials and Methods}

\section{Study Population}

A prospective study of cervical spine MDCT was conducted to compare standard-dose with low-dose examinations in patients with blunt trauma. In all of the low-dose scan protocols, 4D tube current modulation was used, whereby the tube current is adapted according to the $\mathrm{x}-, \mathrm{y}-$, and $\mathrm{z}$-axis of the scan process and this current tube adaption occurs continuously during the scan time. From October 2005 to September 2006, 191 consecutive patients (96 men and 95 women; mean age, 46.8 years; age range, $16-80$ years) were scanned on 2 different CT systems, a 6- and 16-MDCT (Emotion 6 and Sensation 16; Siemens Medical Systems, Erlangen, Germany): 51 patients were scanned using a standard (normal-dose) protocol and 140 were scanned with a low-dose scan protocol.

All of the patients were referred directly from the emergency department with an acute history of blunt trauma. Forty-seven of them were polytrauma patients, most $(n=36)$ with severe craniocerebral trauma with altered mental status or unconsciousness, warranting further investigation with cranial CT. Clinical cervical spine evaluation in the patients was impossible or unreliable. The other patients $(n=119)$ had an anamnesis of neck pain after blunt trauma and showed midline tenderness of the cervical spine at clinical examination. In 25 patients clinical diagnosis was unreliable because of intoxication (uncooperative or incoherent patients) after a motor vehicle crash.

The study protocol was approved by the ethical review board of our institution. The need for individual patient informed consent was waived, because the use of these low-dose MDCT scan protocols had been implemented already for more than 2 years in the evaluation of neck pain in nontrauma patients with good clinical results.

\section{CT Examinations}

All of the patients were examined in the supine position without contrast material. A lateral scout image preceded the helical acquisition, 

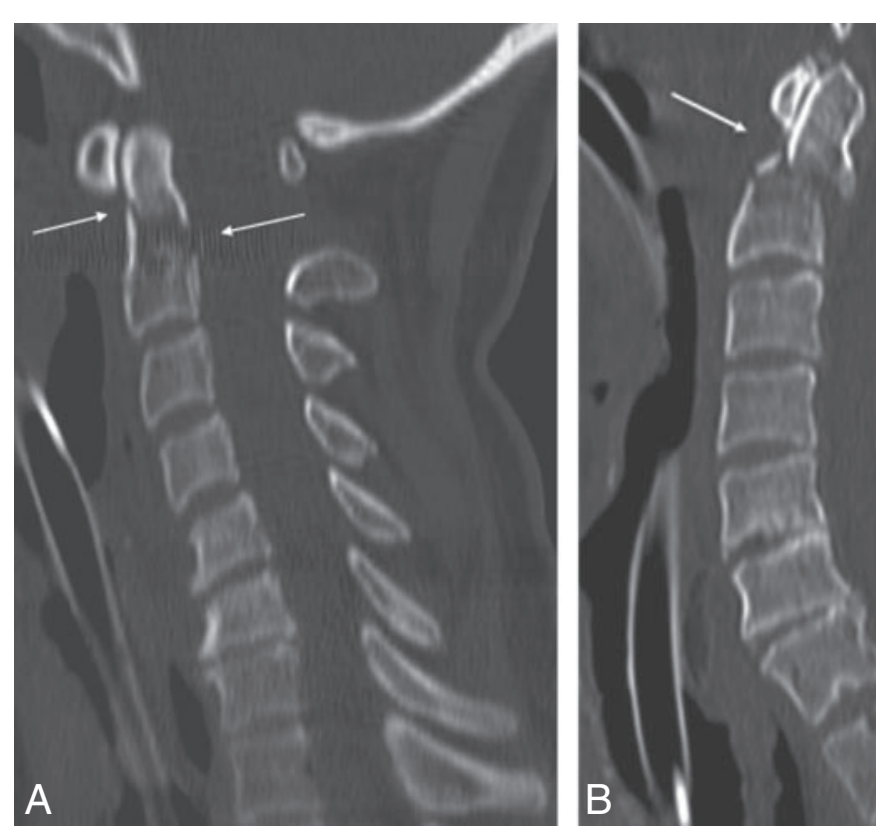

which reached from the foramen magnum to the bottom of the first thoracic vertebral corpus (Th1). For standard-dose examinations, the following scan protocols were used without tube current modulation: 6 -MDCT $(n=30)$ : tube voltage of $130 \mathrm{kV}, 6 \times 1 \mathrm{~mm}$ collimation, 0.6 -second rotation, table feed of $4 \mathrm{~mm}$ per rotation (pitch factor $=$ 0.66 ), and 175 effective tube current-time product ( $\mathrm{mAs}$ ), which gives a volume CT dose index (CTDI vol) of $23.17 \mathrm{mGy}$; 16-MDCT ( $n=$ 21): $120 \mathrm{kV}, 16 \times 1.5 \mathrm{~mm}$ collimation, 0.5 -second rotation, table feed of $24 \mathrm{~mm}$ per rotation (pitch factor $=1$ ), and 250 effective mAs, which gives a CTDI vol of $19.24 \mathrm{mGy}$.

All of the low-dose examinations were done with tube current modulation, but with 2 different tube voltage (kilovolt) settings: because the neck is the smallest part of the body, with exception of the limbs, use of the highest tube voltage of each CT system, as proposed by the manufacturer, seemed unnecessary to achieve adequate image quality for cervical spine evaluation. In low-dose examinations, the following scan protocols were used: 6-MDCT with $130 \mathrm{kV}$ (high $\mathrm{kV}$, $n=28)$ or $110 \mathrm{kV}$ (low kV, $n=28), 175$ "reference" effective mAs and other scan parameters unchanged as in the standard dose protocol. This gives a CTDI vol of $23.17 \mathrm{mGy}(130 \mathrm{kV})$ and $15.27 \mathrm{mGy}(110$ $\mathrm{kV}$ ), 16-MDCT with $120 \mathrm{kV}$ (high $\mathrm{kV}, n=42$ ) or $100 \mathrm{kV}$ (low $\mathrm{kV}, n=$ 42 ), and other scan parameters unchanged as in the standard-dose protocol, which gives a CTDI vol of $19.48 \mathrm{mGy}(120 \mathrm{kV})$ and 12.48 mGy $(100 \mathrm{kV})$.

Overlapping 2-mm-thick images (with 1-mm recon increment) were reconstructed from the raw dataset with a hard (bone) filter algorithm on both CT systems. From this MDCT dataset, consecutive sagittal and coronal images were obtained with 2.5-mm-section thickness. Additional 2-mm axial images were obtained in unequivocal cases $(n=48)$. The tube current modulation system in the lowdose examinations is CareDose4D (Siemens Medical Systems). This on-line tube current modulation combines 2 forms of modulation: in the $\mathrm{z}$-axis and in the $\mathrm{x}-\mathrm{y}$ axis (angular modulation). Tube current modulation in the $\mathrm{z}$-axis is determined from attenuation values and shape obtained by refined analysis of an anteroposterior or lateral projection radiogram at the start of the examination. In z-axis modulation, tube current is adjusted to maintain a user-selected image quality level in the image data. Noise is regulated on the final image to a level desired by the user in an attempt to render all of the images of
Fig 1. A, A 53-year-old woman after polytrauma. Sagittal $2.50 \mathrm{~mm}$ image of standard-dose 16-MDCT examination (120 $\mathrm{kV}$ and fixed $250 \mathrm{mAs}$ ) shows fracture at the base of the dens of C2 (arrow). Calculated effective at examination is $3.9 \mathrm{mSv}$. B, A 62-year-old man after polytrauma. Sagittal 2.5-mm image of low-dose 16-MDCT examination (100 kV and 141 $\mathrm{mAs}$, after tube current modulation) shows fracture at the base of the dens of C2 (arrow). Calculated effective dose is $1.4 \mathrm{mSv}$.

similar noise, independent of patient size and anatomy. ${ }^{14,15}$ In this sense, $\mathrm{z}$-axis modulation is the CT equivalent of the autoexposure control systems used for many years with conventional $\mathrm{x}$-ray systems. ${ }^{15}$ Angular (x-y) tube current modulation works differently: the tube current is adjusted to minimize $\mathrm{x}$-rays in projections (angles) that are of less importance for the reduction of overall image noise content. ${ }^{14-17}$ Noise in CT scans is dominated by those projections in which the attenuation is highest. For a homogeneous object with a circular cross-section, attenuation is constant over all of the projections, and all of the measured values contribute equally. However, for a nonhomogeneous object, like the human body, with a noncircular cross-section, attenuation varies strongly. ${ }^{16,17}$ Because noise measured from high-attenuation projections (ie, lateral direction) greatly influences the noise level in CT data, the dose for projections with relatively low attenuation (ie, anteroposterior direction) can be reduced substantially without a measurable increase in image noise. ${ }^{16,17}$ Angular tube current modulation is characterized by on-line monitoring of the attenuation and subsequent tuning of the tube current as a function of the projection angle with a delay of $360^{\circ}$.

For each section position, the CT system calculates the average tube current, expressed as average effective mAs, throughout the duration of exposure. Effective $\mathrm{mAs}{ }^{18}$ are determined by dividing the product of milliamperes and rotation time by the pitch, which is the ratio between the table feed per rotation and the $\mathrm{x}$-ray beam width or collimation. ${ }^{19}$ The mean effective milliampere seconds of the whole examination are displayed at the scan console at the end of the examination and were recorded in our study. The calculation of the effective dose (millisieverts) was done with commercially available software on a personal computer (WinDose; Institut für Medizinische Physik, Universität Erlangen, Erlangen Germany). ${ }^{20}$ This software does not require phantom measurements, but inputs of the scan parameters (sex, scan region and length, tube voltage, current-time product, pitch, and multidetector nature) are represented on a graph of the Monte Carlo phantom model, and calculation of effective dose is computer simulated according to the recommendations of the International Commission on Radiologic Protection (report ICRP-60).

\section{Evaluation of Image Quality}

Objective evaluation of image quality was based on the evaluation of image noise, by measurements of the SD in CT numbers in Hounsfield units (HUs) for pixels in a standard $1-\mathrm{cm}^{2}$ circular region of interest (region of interest), placed in the same anatomic location for all of the examinations: spinal medulla at level C5 on the midline sagittal $2.5-\mathrm{mm}$ image. This was done by the same radiologist (R1) in all 191 of the examinations.

Subjective evaluation of image quality was performed using a scoring system, for assessing the level of image noise in the images, 

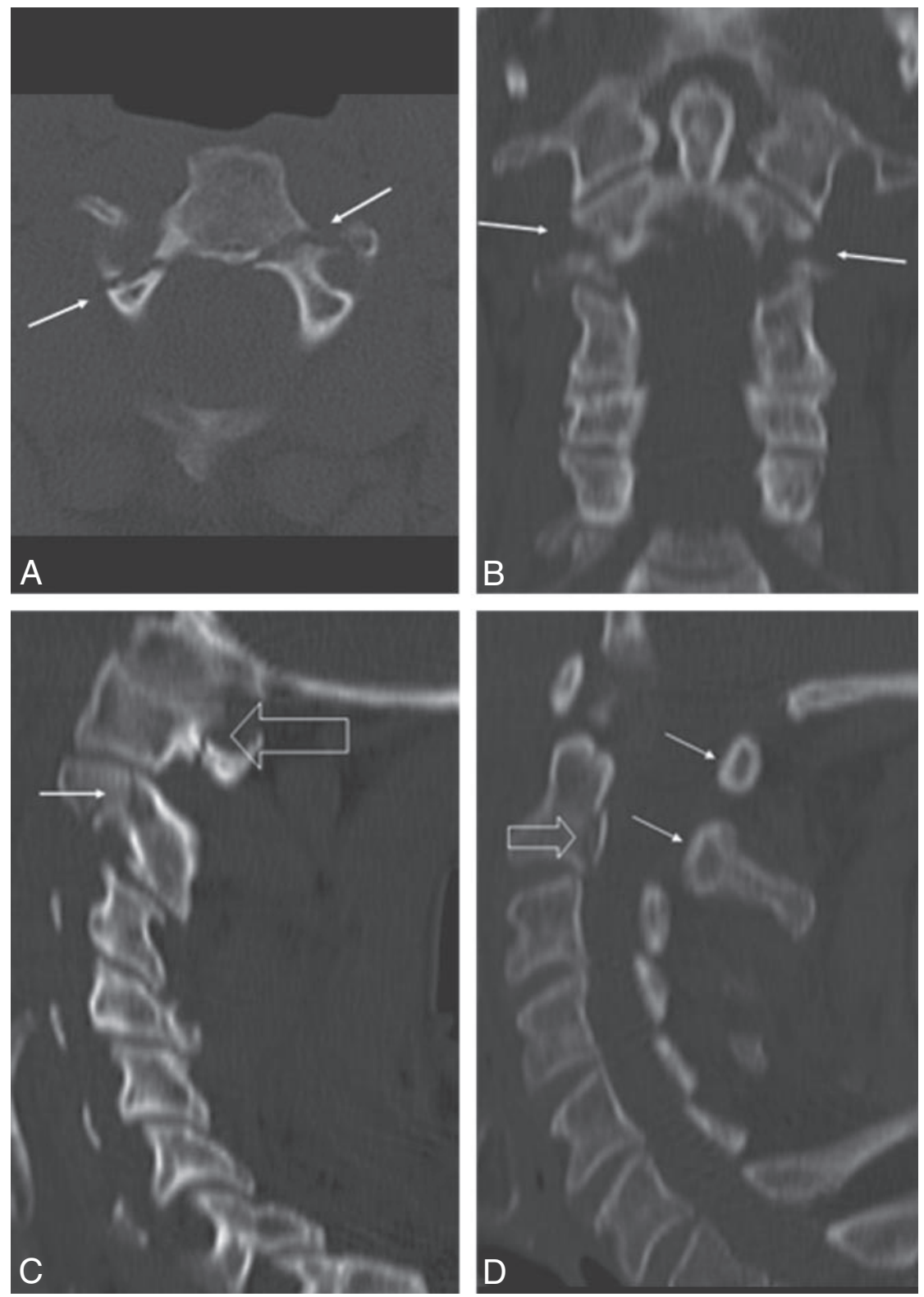

Fig 2. A, A 53-year-old-man after a high-velocity motor vehicle crash with head injury. Axial 2.5-mm image shows double fracture (arrows) at the transition of the body with each posterior arc of C2. Calculated effective dose of lowdose 16-MDCT examination ( $100 \mathrm{kV}$ and $96 \mathrm{mAs}$ ) is $0.98 \mathrm{mSv}$. $B$, Coronal $2.5-\mathrm{mm}$ image shows bilateral fractures at the base of each lateral mass of C2 (arrows). C, Sagittal 2.5-mm image shows fracture at the left arc of $\mathrm{C} 2$ (arrow) and at the left arc of $\mathrm{C} 1$ (open arrow). D, Sagittal 2.5-mm image shows avulsion fracture of the posterior margin of the body of C2 (open arrow) and posterior subluxation of both posterior arches of C1 and C2 (arrows).

second (retrospective) interpretation session was performed: all of the reconstructed images of each CT system (6- and 16-MDCT), were loaded and viewed on a separate Leonardo workstation (Siemens Medical Systems), which included 51 standard- and 140 low-dose examinations. Of these examinations, the consecutive 2.5 -mm-thick sagittal and coronal (and sometimes additional axial) images were examined by 2 reviewers: 2 residents, 1 with 1 year of experience in interpretation of CT (R3) and 1 with no experience in interpretation of CT (R4). The readers were blinded for the scan protocol used (scan parameters were removed) but were aware of the clinical information. They were asked to record the same parameters as in the first image interpretation session: presence of fracture, fracture type, (sub)luxation, and image quality score.

\section{Statistical Analysis}

Statistical analysis was performed with commercially available statistical software (InStat 3.0; GraphPad Software, San Diego, Calif). Comparison of calculated effective dose was done with a Kruskal-Wallis test and comparative post hoc Dunn tests among the 3 patient groups: standard-dose examinations $(n=51)$, low-dose examinations with tube current modulation and high kilovolt $(n=70)$, and

ranking from 0 to 4 ( 4 = excellent image quality: absent or very minimal noise; 3 = good image quality: minimal image noise; $2=$ moderate image quality: moderate image noise, not interfering with diagnostic quality; 1 = bad image quality: too much noise, may interfere with diagnostic quality; and $0=$ very bad image quality: very much noise, not useful for diagnostic imaging), whereby the readers were blinded for the scan parameters (removed in the display). Diagnostic image quality was considered to have been achieved when the score was 2 or higher.

All of the images of each patient were viewed at the day of presentation of the patient using a clinical workstation (Wizard; Siemens Medical Systems) by 2 readers (R1 and R2) with more than 12 years of experience in CT. They were allowed to use all of the workstation functions, including multiplanar reformation, curved reformation, and $3 \mathrm{D}$ possibilities. The reviewers received the clinical information, especially the region of the patient's pain. They were asked to record the presence or absence of fracture, description of fracture type (especially distinction between stable and unstable types), presence of (sub)luxation, and image quality score. After an interval of 8 weeks, a low-dose examinations with tube current modulation and low kilovolt $(n=70)$. Objective image quality scores, that is, the $\mathrm{SD}$ of region of interest measurements in CT number, were compared with a Kruskal-Wallis test with comparative post hoc Dunn tests among the same 3 examination groups.

Of each of the 4 reviewers, the subjective image quality scores were compared among each of the 3 examinations groups with a KruskalWallis test, with comparative post hoc Dunn tests. Statistical significance for all of the tests was set at a $P<.05$.

\section{Results}

\section{CT Diagnosis}

Of 191 patients, MDCT showed 18 patients with fracture(s) (9.4\%), 3 in the standard-dose examinations and 15 in the lowdose examinations, with 4 fractures in the high-kilovolt group and 11 in the low-kilovolt group. Of these 18 fractures, 14 were unstable fractures $(77.7 \%)$. Most fractures were situated at the C1-C2 level $(n=12)$ : a large number of fractures at the base of the dens axis alone $(n=4$; Fig 1$)$ or in combination with other 

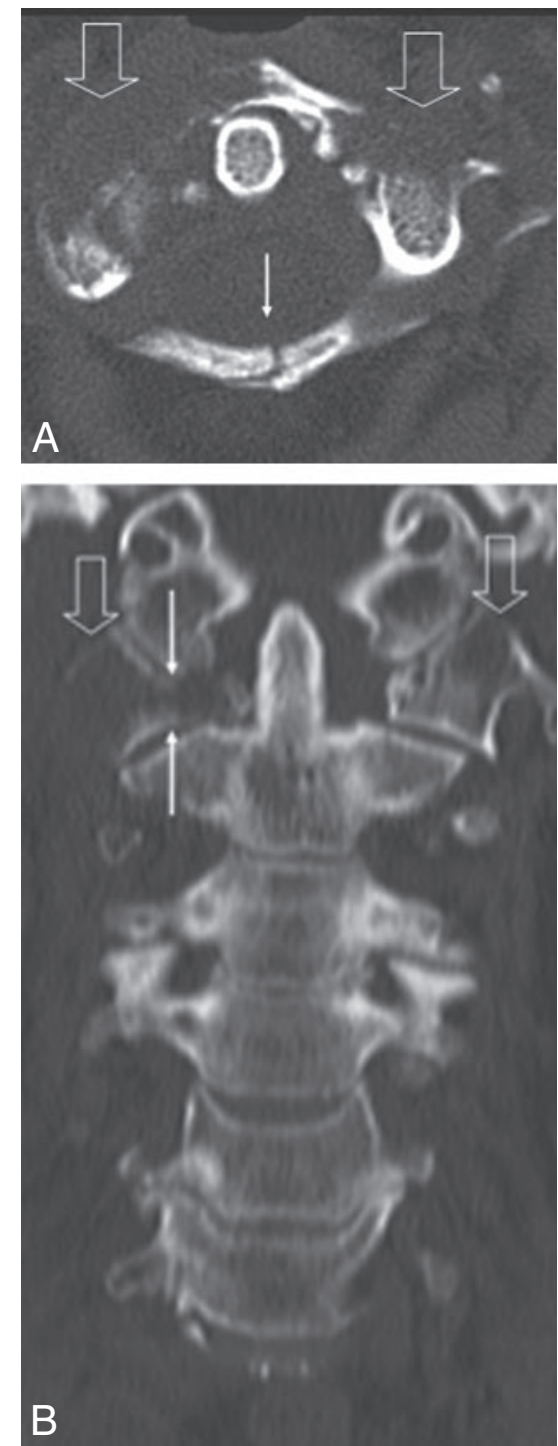

Fig 3. A, A 50-year-old woman with history of lung carcinoma with heavy neck pain after minor blunt cervical trauma. Axial 2.5-mm image shows bilateral osteolytic lesions in each lateral mass of the atlas (C1) (open arrows) and midline fracture (arrow) of the posterior arc of $\mathrm{C} 1$. Calculated effective dose of low-dose 6-MDCT examination (130 kV and $89 \mathrm{mAs}$ ) is $1.48 \mathrm{mSv}$. $B$, Coronal 2.5-mm image confirms bilateral osteolytic lesions in each lateral mass of $\mathrm{C} 1$ lopen arrows) and transverse fracture (arrows) trough the right lateral mass.

fractures $(n=2)$ at the level of C1 or C2 (Fig 2); 3 bilateral fractures of the arcus of $\mathrm{C} 1$ and/or $\mathrm{C} 2 ; 2$ pathologic fractures at the level of C2 (Fig 3); and 1 Hangman fracture. Two patients had an unstable burst fracture of a vertebral body (Fig 4). In 3 fractures, there was associated subluxation. Stable fractures were fractures of a spinous $(n=2$; Fig 5$)$ or of a lateral process $(n=2)$. The 2 experienced radiologists (R1 and R2) correctly identified all of the fractures in the first image interpretation at the moment of the presentation of the patient. The resident with 1 year of experience in CT (R3) also correctly identified all of the fractures. The firstyear resident with no CT experience (R4) missed 3 fractures: 1 unstable fracture at the base of the dens axis (C2) and 2 stable fractures, 1 of a spinous and 1 of a transverse process. In 4 patients, standard radiography of the cervical spine at the moment of the presentation of the patient was interpreted as negative, whereby MDCT clearly depicted a fracture, 2 of them unstable fractures at the base of the dens axis (Fig 6).
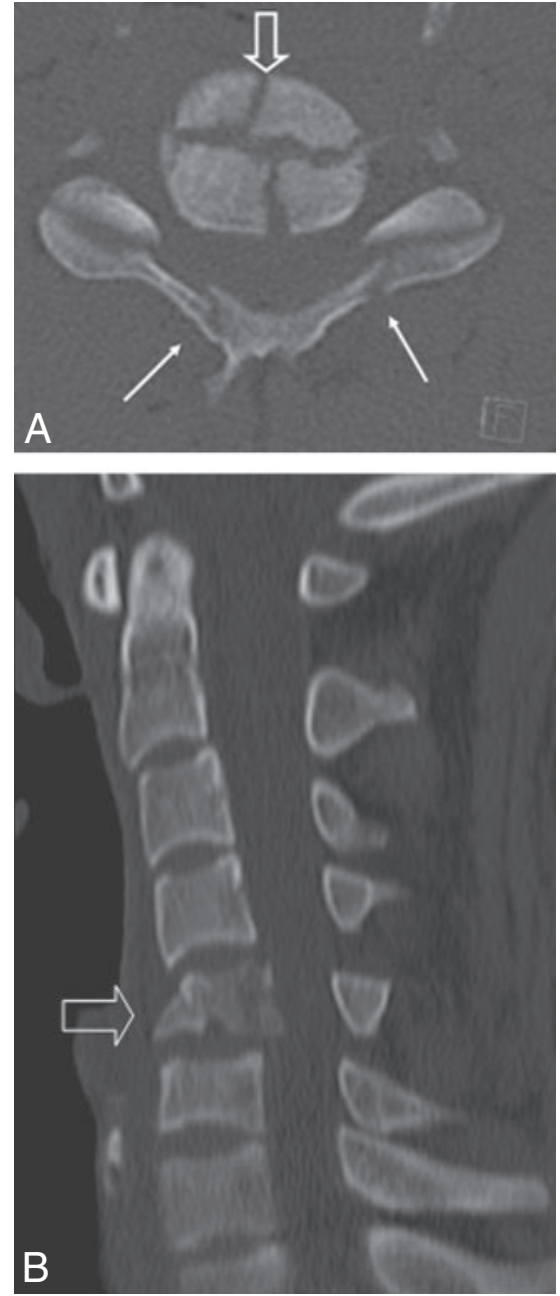

Fig 4. A, A 25-year-old man with tetraplegia after a diving accident. Axial 2.5-mm image shows cross-like burst fracture of the body of $\mathrm{C} 5$ (open arrow) with posterior displacement and bilateral fractures (arrows) of the posterior arcs. Calculated effective dose of low-dose 16-MDCT examination (120 kV and $160 \mathrm{mAs}$ ) is $2.6 \mathrm{mSv}$. B. Sagittal 2.5-mm image shows burst fracture of the body of $\mathrm{C} 5$ (open arrow) with posterior displacement and spinal cord compression.

\section{Dose Reduction}

The use of tube current modulation in cervical spine MDCT of trauma patients gave a substantial dose reduction in comparison with scans with fixed mAs: in our study, expressed in reduction of mean effective mAs, this varies from $50 \%$ to $57 \%$ for the 16 MDCT system to $61 \%$ for the 6-MDCT system. For the $16-$ MDCT system, mean effective mAs lowered from $250 \mathrm{mAs}$ to 106.3 mAs and 125.2 mAs, and for the 6-MDCT system, mean effective mAs lowered from $175 \mathrm{mAs}$ to $68.1 \mathrm{mAs}$ and $67.7 \mathrm{mAs}$ for the low-dose examinations with high and low kilovolts, respectively, and this by use of the tube current modulation alone.

Mean calculated effective dose for the standard dose examinations with fixed $\mathrm{mAs}$ (Table) was $3.75 \pm 0.25 \mathrm{mSv}(\mathrm{SD})$; 16-MDCT system had a mean of $4.01 \pm 0.06 \mathrm{mSv}(\mathrm{SD})$, and 6-MDCT had a mean of $3.57 \pm 0.15 \mathrm{mSv}(\mathrm{SD})$.

Low-dose examinations with tube current modulation and high kilovolts (Table) had a mean calculated effective dose of $1.57 \pm 0.38 \mathrm{mSv}(\mathrm{SD})$ or a mean dose reduction of $58 \%$ compared with standard dose examinations (mean 16-MDCT: $1.7 \pm 0.36 \mathrm{mSv}$ [SD]; mean 6-MDCT: $1.37 \pm 0.33 \mathrm{mSv}$ [SD]). Low-dose examinations with tube current modulation and 


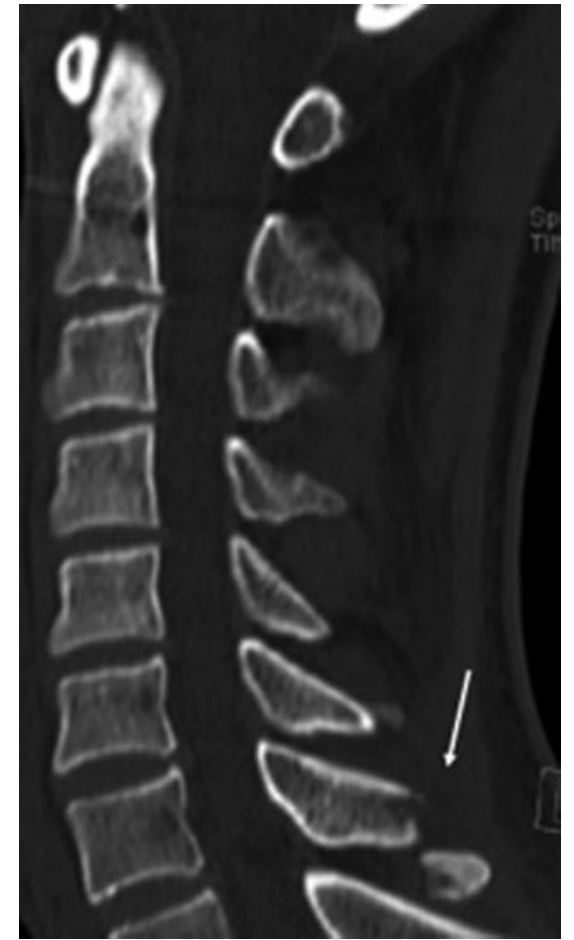

Fig 5. A 39-year-old man with hyperextension trauma of the cervical spine. Sagittal 2.5-mm image shows fracture of the spinous process of $\mathrm{C} 7$ (arrow). Calculated effective dose of low-dose 6-MDCT examination (110 kV and $56 \mathrm{mAs}$ ) is $0.78 \mathrm{mSv}$.

low kilovolts had a mean calculated effective dose of $1.08 \pm$ $0.28 \mathrm{mSv}(\mathrm{SD})$ or a mean dose reduction of $71 \%$ compared with standard dose examinations (mean 16-MDCT: $1.19 \pm$ $0.27 \mathrm{mSv}$ [SD]; mean 6-MDCT: $0.90 \pm 0.29 \mathrm{mSv}$ [SD]).

A Kruskal-Wallis test showed that these dose differences between the standard-dose and the 2 low-dose groups are statistically significant $(P<.0001)$. Comparative post hoc Dunn tests showed a significant difference $(P<.001)$ among pairwise comparison of the 3 examination groups separately. With the low-dose examinations at low kilovolts, a mean effective dose level of approximately $1 \mathrm{mSv}$ is reached, which is approximately 3-4 times the effective dose of a 5-view standard radiography examination $(0.25-0.30 \mathrm{mSv}) .^{21}$

\section{Image Quality Analysis}

Objective image quality analysis, by use of SD of CT numbers, showed a mean noise of $14.82 \pm 5.46 \mathrm{HU}$ (SD) for the standard-dose examinations, $17.46 \pm 6.50 \mathrm{HU}(\mathrm{SD})$ for the lowdose examinations with high kilovolts, and $19.72 \pm 6.17 \mathrm{HU}$ (SD) for the low-dose examinations with low kilovolts (Table). Statistical difference in image noise among the 3 examination groups with a Kruskal-Wallis test was significant $(P=$ .0001). However, comparative post hoc Dunn tests among the standard-dose and the low-dose examinations with high kilovolts and between the low-dose examinations with high and low kilovolts were not statistically significant $(P>.05)$.

Evaluation of subjective image quality with Kruskal-Wallis tests showed no statistically significant difference in image quality score among the 3 examination groups for each of the 4 reviewers: $P$ values were $.61, .32, .18$, and .31, respectively, for R1 to R4. Comparative post hoc Dunn test showed no significant difference (all $P>.05$ ) among pairwise comparison of the 3 examination groups for each reviewer. Mean subjective image quality scores of the 4 reviewers are listed in the Table, whereby all of the reviewers scored a mean value around " 3 " for each of the 3 examination groups, which was considered "good image quality."

In 4 patients, subjective image quality score of 1 of the 4 reviewers was less than 2 (which was considered as the lower limit of acceptable diagnostic image quality): in 1 experienced reviewer (R1) in a standard-dose examination, in 1 reviewer (resident R3) in a low-dose examination with high kilovolts, and 2 in a lowdose examination with low kilovolts (residents R3 and R4).

\section{Discussion}

Prompt identification of CSI in patients with blunt trauma has always been a critical issue in trauma management. Little controversy exists regarding the need for accurate and fast imaging assessment of the traumatized spine to evaluate spinal stability and integrity of the neural elements. ${ }^{2}$

The optimal imaging protocol for cervical spine clearance in patients with blunt trauma, especially in unconscious patients with traumatic brain injury, remains controversial..$^{2-10,22}$ With the proliferation of helical and MDCT, it became clear how poor standard radiography can be for the assessment of cervical spine fractures compared with CT. In a meta-analysis, the percentage of missed fractures on radiography versus CT was found between $1.5 \%$ and $52.0 \%,{ }^{22}$ whereby it is accepted that $10 \%-20 \%$ of significant CSIs are missed by using radiographs. ${ }^{23}$ Despite the absence of a randomized, controlled trial, ample evidence exists that CT outperforms plain radiography as a screening test for patients at high risk of CSI. ${ }^{22}$ Subsequently, CT should be the initial screening test in those patients, especially when there is a significantly depressed mental status. ${ }^{22}$ This has been shown in multiple recent studies ${ }^{3-10}$ : plain radiographs need not to be obtained in those patients, and the Eastern Association for the Surgery of Trauma guidelines ${ }^{24,25}$ for cervical spine evaluation of blunt cervical spine trauma should be updated to reflect this evolving practice pattern. ${ }^{6}$

Clinical evaluation of the neck can reliably rule out significant CSI in the awake and alert blunt trauma patients, so they do not need imaging. ${ }^{2,11,12,24-26}$ There is not sufficient evidence to suggest that cervical spine CT should replace plain radiography as the initial screening test for less injured patients who are at low risk for CSI but still require a screening radiographic procedure. ${ }^{22,23}$ Even in the patients who are at moderate-to-high risk, only a small number will have a significant CSI: there is only a $2 \%-6 \%$ incidence of significant cervical spinal injury in the symptomatic population after acute trauma. ${ }^{2,23-25}$ MDCT of the whole cervical spine is probably becoming a routine screening tool in a significant number of CSI patients, those who are at moderate and high risk. This will lead to a significant increase in workload for radiology departments and in radiation exposure to the population. ${ }^{4,13,23}$ Furthermore, with the introduction of MDCT, there is a clinical trend to increased use for complete examination of the cervical spine with CT in trauma patients, and this changing trend results in a significantly higher radiation dose in a population where most are young and otherwise healthy persons. ${ }^{13}$

There are only a few studies that stress the attention on radiation exposure of CT in evaluation of patients with cervical spine trauma. ${ }^{4,7,23}$ In a recent study, a very high mean effective dose of $26 \mathrm{mSv}$ was reached with cervical spine helical 

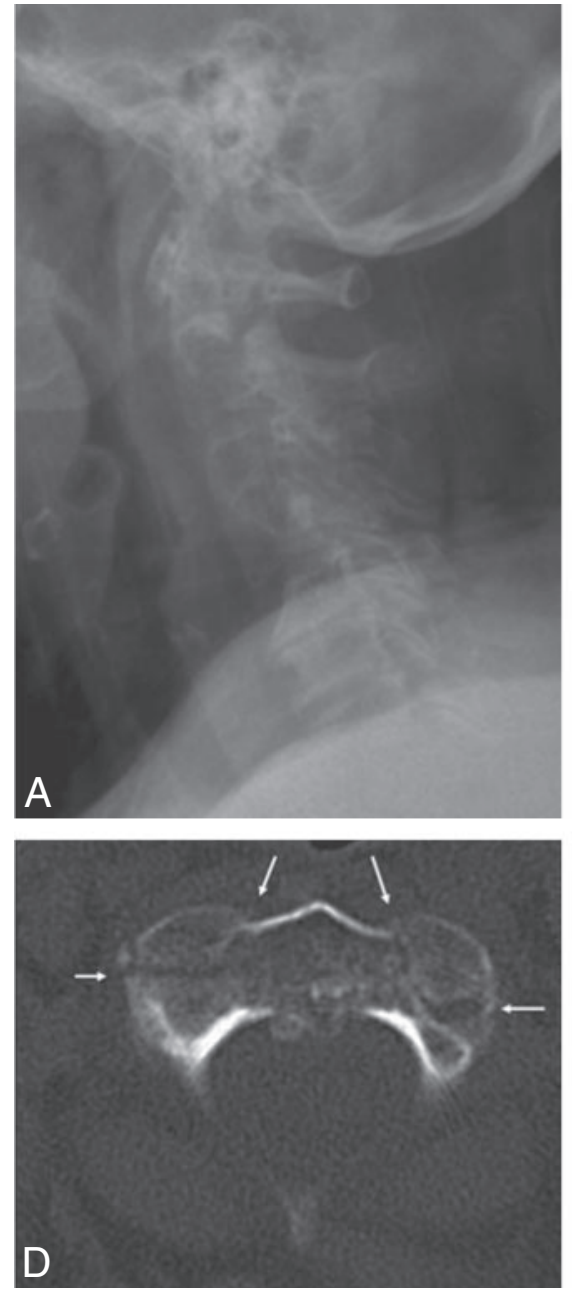
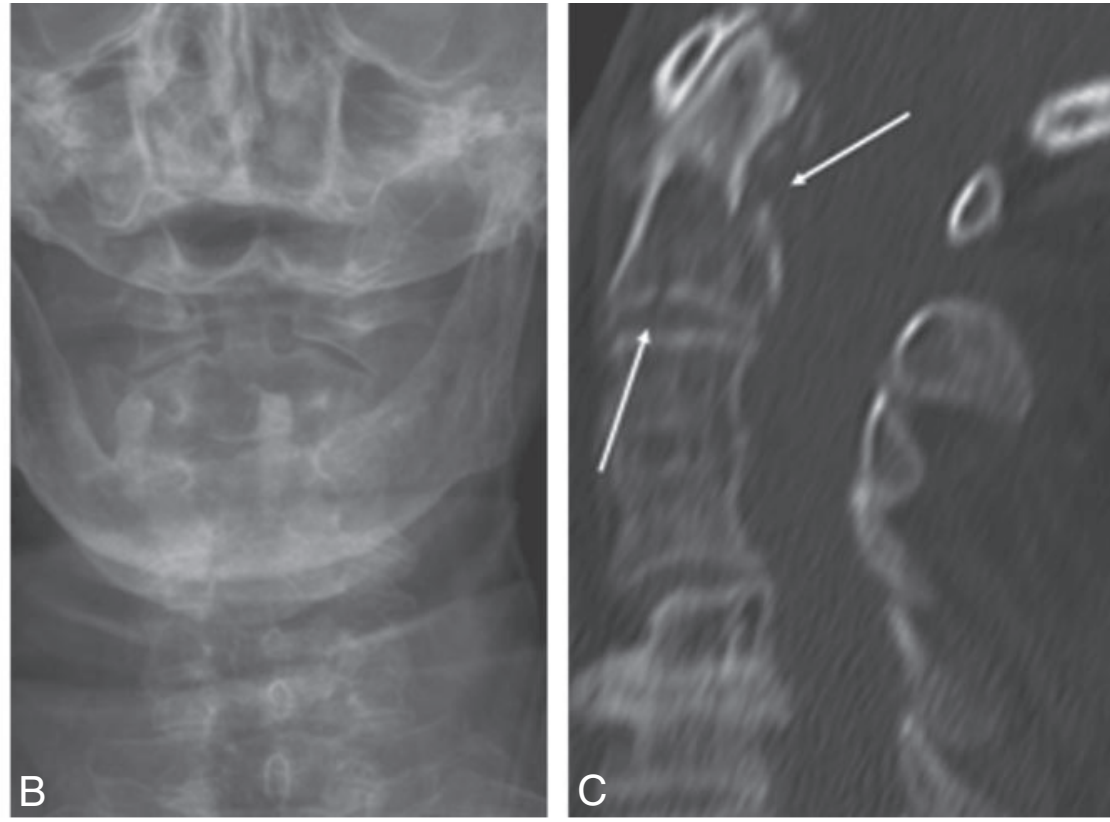

Fig 6. $A$, An 82-year-old woman with acute neck pain after a motor vehicle crash. Standard radiography, lateral view, was interpreted as negative, but additional CT was proposed because of technical failure to view the lower cervica segment C7 (superposition of the shoulders). B, Standard radiograph with odontoid view was interpreted as negative for

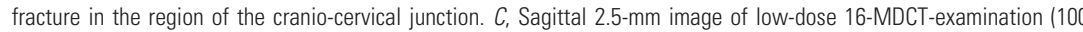
$\mathrm{kV}$ and $165 \mathrm{mAs}$ ) clearly depicts fracture (arrows) at the base of the axis (C2). D, Axial 2.5-mm image of the same low-dose CT examination shows more complex fracture of the body of C2, bilaterally extending in the lateral masses (arrows). Calculated effective dose of MDCT examination is $1.3 \mathrm{mSv}$.

\begin{tabular}{lccc}
\hline \multicolumn{4}{l}{ Data of standard- and low-dose cervical spine trauma MDCT examinations } \\
\hline Variable & Standard Dose & Low Dose: High Tube Voltage & Low Dose: Low Tube Voltage \\
\hline Tube type & Fixed tube current & Tube current modulation & Tube current modulation \\
No. of patients & 51 & 70 & 70 \\
Effective dose, mSv* & $3.75 \pm 0.25$ & $1.57 \pm 0.38$ & $1.08 \pm 0.28$ \\
Image noise, HU* & $14.82 \pm 5.46$ & $17.46 \pm 6.50$ & $19.72 \pm 6.17$ \\
Image quality scores (0-4)* & & & \\
$\quad$ Reviewer 1 & $3.02 \pm 0.62$ & $3.04 \pm 0.51$ & $2.95 \pm 0.65$ \\
Reviewer 2 & $3.02 \pm 0.47$ & $3.06 \pm 0.52$ & $2.94 \pm 0.56$ \\
Reviewer 3 & $2.92 \pm 0.64$ & $2.99 \pm 0.63$ & $2.81 \pm 0.62$ \\
Reviewer 4 & $2.82 \pm 0.65$ & $2.90 \pm 0.71$ & $2.70 \pm 0.67$ \\
\hline
\end{tabular}

Note:-Reviewers 1 and 2 are experienced radiologists; reviewers 3 and 4 are a second-year and a first-year resident, respectively.

* Data are mean \pm SD.

$\mathrm{CT},{ }^{4}$ which is approximately $80-100$ times higher than the radiation dose of a 5-film standard radiographic study $(0.25-$ $0.30 \mathrm{mSv}) .{ }^{21}$ Our study gives a much lower radiation exposure of approximately 4-mSv effective dose with the standard MDCT protocol with fixed tube current, which is approximately 16 times higher than for radiography. With the use of tube current modulation and lower tube voltage, we reached a dose reduction of $71 \%$ without significant difference in image quality evaluation by 4 radiologists and with only a small increase in image noise. A mean effective dose of approximately $1 \mathrm{mSv}$ was achieved, which is only approximately 3-4 times higher compared with standard radiography radiation.

In trauma patients, use of MDCT is first oriented toward fracture and dislocation detection (bone window settings), but reconstruction of images by using soft-tissue filters allows CT to identify some soft-tissue lesions, such as disk herniation and soft-tissue hematomas. ${ }^{2}$ Although MDCT is very sensitive to fracture and subluxation detection, CT is not sensitive to exclusion of soft-tissue trauma, like unstable ligamentous injury, and CT misses spinal cord lesions. ${ }^{2,10,23}$ Cervical MR imaging is appealing, because it detects ligament, disk, and cord injury, but MR imaging also increases cervical clearance time, increases the associated risk with complex patient transport, and is, therefore, not an ideal screening tool. . $^{2,10,23}$

Cervical spine MR imaging is mandatory if the patient with blunt trauma has a neurologic deficit that may be referable to a CSI. ${ }^{2,23,24,25}$ However, patients with blunt trauma with normal neurologic (motor) examination results and normal cer- 
vical spine MDCT findings do not require further radiologic evaluation with MR imaging, before cervical spine clearance, according to a recent study. ${ }^{27}$ In addition, in obtunded and/or "unreliable" patients with blunt trauma, in whom CSI could not be excluded clinically, a normal MDCT of the cervical spine enabled the exclusion of unstable injuries on the basis of follow-up cervical spine MR imaging. ${ }^{28}$

There are several drawbacks in our study. First, there is a relatively low number of patients $(n=191)$ and especially a low number of positive patients: only 18 patients presented with fracture(s) or fracture(s) with subluxation in a 1-year period of screening patients with blunt trauma with possible CSI, who presented at the emergency department of our general hospital. We did no long-term follow-up of our patients, so we have no idea about the possible missed fractures or injury in the acute setting with our MDCT protocols. We did not compare the results of the use of MDCT with other imaging techniques, standard radiography and/or MR imaging. Since the introduction of MDCT in our department in 1998, when a technically good MDCT examination of the cervical spine is performed, additional standard radiography is not routinely requested by the emergency clinicians, especially not in critical or unstable patients or in patients with significant traumatic brain injury, thereby reducing examination time and eliminating additional patient transport and manipulation from the CT to the radiology room. In this way, we treated MDCT as the "gold standard" in our study for the detection of CSI: the use of the CT data as the "gold standard" represents a false end point for the true variable of clinically relevant CSI.

In the second reader session, performed by 2 residents, only sagittal and coronal 2.5-mm reconstructions were reviewed (and sometimes additional axial images, $n=48$ ). This method of evaluation of vertebral fractures was validated in a recent study ${ }^{29}$ : reading of sagittal and coronal multiplanar reformations of MDCT is a feasible approach for correct assessment of vertebral fractures and classifying them into stable or unstable; transverse images must only be analyzed in complex fractures or uncertain findings. In this way, cine viewing, curved reformation, and 3D imaging were limited in the second reader session. Although this seemed to be no problem for the diagnostic accuracy of the resident (R3) with some experience in $\mathrm{CT}$, these extra resources could have helped the inexperienced resident (R4) in the diagnosis of cervical spine fractures.

\section{Conclusion}

MDCT of the whole cervical spine will probably become the first screening tool in place of standard radiography for exclusion of CSI in patients who require imaging after blunt trauma, especially in the patients at high risk and in unconscious patients. This leads to a significantly higher radiation dose in those patients, of whom most are young patients who will have negative findings. Our study shows that, in modern MDCT, the use of tube current modulation and lower tube voltage settings can significantly reduce this radiation dose, compared with standard dose MDCT with fixed tube current, thereby preserving adequate image quality for the detection of cervical spine fractures.

\section{References}

1. Linton OW, Mettler FA; National Council on Radiation Protection and Measurements. National conference on dose reduction in CT, with an emphasis on pediatric patients. AJR Am J Roentgenol 2003;181:321-29
2. Van Goethem JW, Maes M, Özsarlak Ö, et al. Imaging in spinal trauma. Eur Radiol 2005; 15:582-90

3. Griffen MM, Frykberg ER, Kerwin AJ, et al. Radiographic clearance of blunt cervical spine injury: plain radiography or computed tomography scan? J Trauma 2003;55:222-26

4. Antevil JL, Sise MJ, Sack DI, et al. Spiral computed tomography for the initial evaluation of spine trauma: a new standard of care? J Trauma, 2006;61:382-87

5. Widder S, Doig C, Burrowes P, et al. Prospective evaluation of computed tomography scanning for the spinal clearance of obtunded trauma patients: preliminary results. J Trauma 2004;56:1179-84

6. Gale SC, Gracias VH, Reilly PM, et al. The inefficiency of plain radiography to evaluate the cervical spine after blunt trauma. J Trauma 2005;59:1121-25

7. Diaz JJ Jr, Gillman C, Morris JA Jr, et al. Are five-view plain films of the cervical spine unreliable? A prospective evaluation in blunt trauma patients with altered mental status. J Trauma 2003;55:658-63

8. Sanchez B, Waxman K, Jones T, et al. Cervical spine clearance in blunt trauma: evaluation of a computed tomography-based protocol. J Trauma 2005;59:179-83

9. Brohi K, Healy M, Fotheringham T, et al. Helical computed tomographic scanning for the evaluation of the cervical spine in the unconscious, intubated trauma patient. J Trauma 2005;58:897-901

10. Cooper DJ, Ackland HM. Clearing the cervical spine in unconscious head injured patients - the evidence. Crit Care Resusc 2005;7:181-84

11. Grossman MD, Reilly PM, Gillet T, et al. National survey of the incidence of cervical spine injury and approach to cervical spine clearance in U.S. trauma centers. J Trauma 1999;47:684-90

12. Hoffman JR, Mower WR, Wolfson AB, et al. Validity of a set of clinical criteria to rule out injury to the cervical spine in patients with blunt trauma. National Emergency X-Radiography Utilization Study Group. N Engl J Med 2000;343:94-99

13. Chan PN, Antonio GE, Griffith JF, et al. Computed tomography for cervical spine trauma. The impact of MDCT on fracture detection and dose deposition. Emerg Radiol 2005;11:286-90

14. Mulkens TH, Bellinck $\mathrm{P}$, Baeyaert $\mathrm{M}$, et al. Use of an automatic exposure control mechanism for dose optimization in multi-detector row CT examinations: clinical evaluation. Radiology 2005;237:213-23

15. Kalra MK, Maher MM, Toth TL, et al. Strategies for CT radiation dose optimization. Radiology 2004;230:619-28

16. Kalender WA, Wolf H, Suess C, et al. Dose reduction in CT by on-line tube current control: principles and validation on phantoms and cadavers. Eur Radiol 1999;9:323-28

17. Greess $\mathrm{H}$, Wolf $\mathrm{H}$, Baum $\mathrm{U}$, et al. Dose reduction in computed tomography by attenuation-based on-line modulation of tube current: evaluation of six anatomical regions. Eur Radiol 2000;10:391-94

18. Mahesh M, Scatarige JC, Cooper J, et al. Dose and pitch relationship for a particular multislice CT scanner. AJR Am J Roentgenol 2001;177:1273-75

19. Silverman PM, Kalender WA, Hazle JD. Common terminology for single and multislice helical CT. AJR Am J Roentgenol 2001;176:1135-36

20. Kalender WA, Schmidt B, Zankl M, et al. A PC program for estimating organ dose and effective dose values in computed tomography. Eur Radiol 1999;9:555-62

21. United Nations Scientific Committee on the Effects of Atomic Radiation (UNSCEAR). Sources, Effects and Risks Of Ionizing Radiation, Vol. 1: Sources. UNSCEAR 2000 Report to the General Assembly, with Scientific Annexes. New York: United Nations Publishing; 2000

22. Holmes JF, Akkinepalli R. Computed tomography versus plain radiography to screen cervical spine injury: a meta-analysis. J Trauma 2005;58:902-05

23. Tins BJ, Cassar-Pullicino VN. Imaging of acute cervical spine injuries: review and outlook. Clin Radiol 2004;59:865-80

24. Pasquale M, Fabian TC. Practice management guidelines for trauma from the Eastern Association for the Surgery of Trauma. EAST ad hoc committee on guideline management: identifying cervical spine instability after trauma. J Trauma 1998;44:941-56

25. Marion D, Domeier R, Dunham CM, et al. Determination of Cervical Spine Stability In Trauma Patients (Update of the 1997 EAST Cervical Spine Clearance Document). 2000. Available at: www.east.org. Accessed September 1, 2005.

26. Gonzalez RP, Fried PO, Bukhalo M, et al. Role of clinical evaluation in screening for blunt cervical spine injury. J Am Coll Surg 1999;189:152-57

27. Schuster R, Waxman K, Sanchez B, et al. Magnetic resonance imaging is not needed to clear cervical spines in blunt trauma patients with normal computed tomographic results and no motor deficits. Arch Surg 2005;140:762-66

28. Hogan GJ, Mirvis SE, Shanmuganathan K, et al. Exclusion of unstable cervical spine injury in obtunded patients with blunt trauma: is MR imaging needed when multi-detector row CT findings are normal? Radiology 2005;237:106-13

29. Begemann PG, Kemper J, Gatzka C, et al. Value of multiplanar reformations (MPR) in multidetector CT (MDCT) of acute vertebral fractures: do we still have to read the transverse images? J Comput Assist Tomogr 2004; 28:572-80 